\title{
The critical phonon scattering and peculiarities of the transport phenomena in ferroelectric crystal
}

\author{
V.I.Altukhov ${ }^{1}$, B.A.Strukov ${ }^{2}$ \\ 1 North-Caucasian State Technical University, 355000 Stavropol, Russia \\ 2 Moscow State University, 119992 Moscow, Russia
}

Received August 6, 2002

\begin{abstract}
The review of the theoretical study of the critical phonon scattering and anomalous heat transport near the phase transition temperature $T_{\mathrm{c}}$ is presented. An interpretation and quantitative approach to the description of the anomalous temperature dependence of the thermal conductivity $\lambda(T)$ near $T_{\mathrm{c}}$ are discussed.
\end{abstract}

Key words: ferroelectrics, phase transitions, critical phonon scattering, heat transport theory, soft-phonon mode, central peak

PACS: 77.84.-s, 77.80.Bh, 63.20.-e, 66.70.+f

\section{Introduction}

The heat conductivity coefficient (HCC) $\lambda(T)$ of ferroelectric crystals shows a sufficient change near the phase transition temperature $T_{\mathrm{c}}[1-3]$. On the curve $\lambda(T)$ may be observed:

(a) a broad minimum-negative peak,

(b) broken or cusp-like negative (positive) anomalies,

(c) step-like anomalies (jumps),

(d) a positive (negative) more or less symmetrical peak and

(e) a point gap of the second order at $T \rightarrow T_{\mathrm{c}} \pm 0$.

In the theory [4-8] usually (a), (b) and (c)-type anomalies are connected with the inelastic scattering of acoustic phonons by the critical ones (soft-TO-phonon mode) or with the quasi-elastic scattering of thermal acoustic phonons by the central peak, which reveals itself in the spectra of scattering light and critical neutron scattering 
at $T \rightarrow T_{\mathrm{c}}[9,10]$. On the whole four mechanisms are known for the critical phonon scattering: (1) the inelastic scattering for $\Gamma_{0} \ll \omega$ (where $\Gamma_{0}$ is the critical damping of the soft mode) [6]; (2) the relaxation mechanism actual for $\omega \leqslant \Gamma_{0}[21,22]$; (3) the quasi-elastic scattering by the central peak $(\omega \rightarrow 0)$ [7]; (4) the interaction of the critical fluctuations with defects $[23,14]$. In this paper the general theory of the most actual mechanism (inelastic and quasi-elastic) of critical phonon scattering and the peculiarities of lattice thermal conductivity for $T \rightarrow T_{\mathrm{c}}$ is developed. The results of the numerical model calculations are compared with the corresponding experimental data.

The phonon Green functions of the ferroelectric crystals with hydrogen bonds or in the ferroelectric ferromagnetics and in the crystal with paramagnet impurities are described in Kobayashi's model for the pseudospin and phonon systems of the crystal [11-14]. The retarded Green function can be found in the hydrodynamics approximation $(\omega \tau \ll 1)$ and the heat conductivity is calculated in Kubo formalism near the structural phase transition temperature $T_{\mathrm{c}}$.

The goal of this work is to show the possible approach to the explanation of the pointed variety of the HCC temperature dependences near $T_{\mathrm{c}}$ taking into account the most realistic mechanisms of the phonon scattering.

\section{The thermal conductivity of crystals}

The general expression for the thermal conductivity $\lambda(T)$ in cubic crystal may be written as Laplace integer $[15,16]$ from heat current correlator

$$
\lambda(T)=\frac{1}{3 V k_{\mathrm{B}} T^{2}} \lim _{\gamma \rightarrow+0} \int_{0}^{\infty} \mathrm{e}^{-\gamma t} \cdot\langle j(t) \cdot j(0)\rangle \mathrm{d} t,
$$

here $k_{\mathrm{B}}$ is the Boltzmann constant. It is shown in $[16,17]$ that in the one-particle approximation the thermal conductivity coefficient $\lambda(T)$ may be expressed by the dynamical Green function $G_{\nu}(\omega), \nu=(k, s), k \equiv \vec{k}$ is the wave vector, and $s$ is the branch index of the phonon with the frequencies $\omega$.

The density heat current $j(r, t)$ determined from the equation

$$
\frac{\partial H(r, t)}{\partial t}+\operatorname{div} j(r, t)=0
$$

where local density energy $(r=\vec{r})$

$$
H(r)=\sum_{l} H(l) \delta\left(R_{l}-r\right)=\frac{1}{\sqrt{V}} \sum_{q} H(q) \mathrm{e}^{-\mathrm{i} q r}
$$

$V=V_{0} N$ is the volume of the crystal $\left(V_{0}=a^{3}\right), l$ is a numbers of the unit cells $(l=1,2 \ldots N), R_{l}=\vec{R}(l), q$ is the quasi-momentum of the phonons. Taking into 
account a Heisenberg movement equation for $H(q, t)$ the heat current operator can be obtained as

$$
\sum_{\alpha} q_{\alpha} j_{\alpha}(q)=\frac{1}{\hbar}[H(q, t), H]
$$

where $\alpha$ is the Cartesian components, $\sum_{r} H(r)$ is the Hamiltonian of the system. Taking into account (1)-(4) and the fact that $\operatorname{Im} G_{\nu}(\omega)$ has a maximum in the vicinity of $\omega^{2}=\omega_{\nu}^{2}$, we obtain the usual gas formula or $\tau$-approximation (when the transport relaxation time $\tau_{T}$ for two-phonon excitement can be approximated by one-phonon relaxation time $\tau$ ) [17]

$$
\lambda(T)=\frac{1}{3 V} \sum_{\nu} \frac{\left(\hbar \omega_{\nu}\right)^{2}}{k_{\mathrm{B}} T^{2}}\left(\nabla_{k} \omega_{\nu}\right)^{2} \frac{n_{\nu}\left(n_{\nu}+1\right)}{\Gamma_{\nu}\left(\omega_{\nu}\right)},
$$

where $n_{\nu}$ is the phonon numbers occupation, $n_{\nu}\left(n_{\nu}+1\right) \hbar^{2} \omega^{2} / k_{\mathrm{B}} T^{2}=C\left(\omega_{\nu}\right)$ is the single mode specific heat, and $\Gamma_{\nu}(\omega)=\tau^{-1}(\omega)$ is an inverse one phonon relaxation time, $\nabla_{k} \omega_{\nu}$ is the group velocity of phonons.

\section{The critical inelastic and quasi-elastic scattering}

\subsection{The critical inelastic scattering}

The inverse relaxation time of acoustic phonons in a crystal with a weak cubic anharmonicity $\Phi(\nu, 1,2)$ can be written as [16]

$$
\Gamma_{\nu}(\omega)=\frac{\pi \hbar}{2} \sum_{1,2}|\Phi(\nu, 1,2)|^{2} \rho_{2}\left(\omega-\omega_{1}\right) \cdot \frac{\Delta \cdot(k-2-1)}{\omega \omega_{1}} \cdot \frac{n\left(\omega-\omega_{1}\right)}{n(\omega)},
$$

where $\pi \rho_{\nu}(\omega)=\operatorname{Im} G_{\nu}(\omega), G_{\nu}(\omega)$ is the Green function of the crystal, $1 \equiv \nu_{1}=$ $\left(k_{1}, s_{1}\right), 2=\nu_{2}, n_{\nu} \equiv n\left(\omega_{\nu}\right)=\left[\exp \left(\hbar \beta \omega_{\nu}\right)-1\right]^{-1}, \beta=1 / k_{\mathrm{B}} T$.

Near the structural phase transition temperature $T_{\mathrm{C}}$ the soft mode (for $q \equiv$ k) $\omega_{q}(T) \rightarrow \omega_{0}\left(T_{0}\right)$ and the spectral density $\rho_{2}(\omega)$ for the critical (soft) branch of vibration $2=\nu_{2}=(q, s)$ determined from a transport equation [8,17], can be approximated as

$$
\pi \rho_{q}(\omega)=\frac{\omega \Gamma_{0}(T)}{\left[\omega_{q}^{2}(T)-\omega^{2}\right]^{2}+\omega^{2} \Gamma_{0}^{2}(T)}+\frac{1}{\omega_{\mathrm{c}}^{2}(q)} \cdot \frac{\omega \gamma_{0}(q)}{\omega^{2}+\gamma^{2}(q)}
$$

Here $\omega_{\mathrm{c}}^{2}(q)=\omega_{q}^{2}(T)-\delta^{2}(T), \gamma(q)=\gamma_{0} \omega_{\mathrm{c}}^{2} / \delta_{0}^{2}, \quad \delta_{0}=\delta\left(T_{\mathrm{C}}\right), \quad \omega_{\mathrm{c}}(q=0$, $\left.T=T_{\mathrm{C}}\right)=0, \quad T_{0} \neq T_{\mathrm{C}}, \quad \gamma(q)$ is the line width of the central peak, and $\gamma_{o}^{-1}$ is the average lifetime of thermal phonons. Substituting (7) into (6) we get

$$
\tau_{\nu}^{-1}(\omega, T)=\tau_{\text {in }}^{-1}(\omega, T)+\tau_{\mathrm{c}}^{-1}(\omega, T),
$$

where $\tau_{\text {in }}^{-1}$ is conditioned by inelastic, and $\tau_{\mathrm{c}}^{-1}$ by quasi-elastic phonon scattering.

Inelastic scattering is more actual in the region of $\omega \ll \omega_{0}=\omega_{0}(T)$ and plays an important role when the critical branch approaches the acoustic one and the 
degeneration of branches takes place. In this case $\vec{q}_{1}-\vec{q}_{2}=\vec{q}$ and assuming that $\omega_{1}=\omega_{0}\left(q_{1}\right), \omega_{2}=\omega_{0}\left(q_{2}\right)=\omega_{0}\left(q_{1}-q\right)$ and considering the fact that $\omega_{\nu} \ll \omega_{0} \ll k_{\mathrm{B}} T$ we obtain

$$
\omega_{0}\left(q_{1}-q\right)-\omega_{0}\left(q_{1}\right) \approx r \omega_{\nu} \cos \Theta
$$

where $r=v_{0} / v$ is the ratio of the mean velocities for critical and acoustic phonon with $\omega_{\nu}=v q$ and $\Theta$ is the angle between $\vec{q}_{1}$ and $\vec{q}_{2}$.

If we neglect the damping of the soft mode $\left(\Gamma_{0} \ll \omega\right)$, then in accordance with $(8)-(6)$

$$
\tau_{\text {in }}^{-1}(\omega)=\frac{\pi q B}{\beta} \cdot \frac{\omega}{\omega_{0}},
$$

where $B$ is a numerical coefficient.

In the case $\Gamma_{0} \gg \omega$ for $\tau_{\text {in }}^{-1}$ one obtains the relaxation formula

$$
\tau_{\text {in }}^{-1}(\omega)=\frac{\pi A \omega}{\beta \omega_{0}} \cdot \frac{\omega \tau_{0}}{1+\left(\omega \tau_{0}\right)^{2}},
$$

The critical damping of the soft mode $\Gamma_{0}=\tau_{0}^{-1}=\Gamma_{0}(\tau)$ was calculated in microscopy theory [18] and was obtained in scaling form $\Gamma_{0}(\tau) \sim \tau^{-\lambda}$, where $\tau=$ $\left|1-T / T_{\mathrm{C}}\right|$ and $\lambda=\nu=1 / 2$ is the critical index of the correlation length $\xi(\tau) \sim \tau^{-1 / 2}$.

\subsection{Quasi-elastic phonon scattering}

At $T \rightarrow T_{\mathrm{C}}$ and $q \rightarrow 0, \omega_{\mathrm{c}}\left(0, T_{\mathrm{C}}\right)=0, \omega=\omega_{\nu} \approx \omega_{2}$ and quasi-elastic scattering becomes actual in (7). In the vicinity of $q=0$ the dispersion of the soft branch may be expressed in the form $\omega_{q}(T)=D\left(q_{0}^{2}+q^{2}\right)$, where $D$ is a temperatureindependent constant, whereas $q_{0}$ depends on temperature and vanishes at $T=T_{0}$. Let $\omega_{1}=\omega\left(q-q_{2}\right)$ and $\omega_{\nu}$ belongs to some acoustic branch $\left(s_{1}=s\right)$. Then, expressing the coupling coefficient $|\Phi(\nu, 1,2)|^{2}$ in the form $\Phi^{2} \omega_{\nu}^{4}$, after some calculations we find

$$
\tau_{\mathrm{c}}^{-1}(\omega)=\frac{a^{3} \Phi^{2}}{8 \beta \pi^{2} \gamma^{2}} \cdot \frac{\omega^{2} \gamma_{0}}{\omega_{0} \delta_{0}} \cdot \frac{\pi}{2 \mu} \cdot \ln \left(1+\frac{q_{m}^{2}}{k^{2}}\right) \cong \frac{\pi A}{\beta} \cdot \frac{\omega^{2} \gamma_{0}}{\delta_{0}^{2} \mu} \cdot \ln \xi(\tau),
$$

where $\mu=2 D \gamma_{0} / v \delta_{0}, q_{m}=\pi / a, A=a^{3} \Phi^{2} / 16 \pi^{2} v^{2}, \xi(\tau)=k^{-1}$ is the correlation length $k^{2}=c \tau, \tau=\left|1-T / T_{\mathrm{C}}\right|, c$ is the constant connected with the dispersion parameter $D$.

Then we find from (10) and (9) that $\tau_{\text {in }} / \tau_{\mathrm{c}} \sim \ln (\tau)$ and it is seen that the quasi-elastic scattering becomes dominant at $T \rightarrow T_{\mathrm{C}}$.

The expressions (10), (11) for inverse relaxation time will be used for numerical calculations of the temperature dependence of the thermal conductivity coefficient $\lambda(T)$ for some types of ferroelectric crystals. Therefore, it is possible in principle to compare this theory with the appropriate experimental data.

\section{Some applications and discussion}

It is well known that even approximate solution of the problem (the one-particle $\tau$-approximation of transport relaxation time) is rather complicated. For quantitative analysis one can use the simple model of the crystal with the Debye-like spectral 
density $\rho\left(\omega^{2}\right)=3 \omega^{2} / \omega_{\mathrm{D}}^{3}, \omega_{\mathrm{D}}=k_{\mathrm{B}} \Theta / \hbar, \Theta$ is the Debye temperature. Let us consider, for example, the most simple model of a crystal with a structural phase transition and with only acoustic, hard optical and soft optical vibrational branches. One can consider the heat flow as a sum of the heat flows carried by each separate mode. Therefore the expression for $\lambda(T)$ according to (5) may be written as

$$
\lambda(T)=\frac{1}{a_{0}^{3}} \int \mathrm{d} \omega^{2} C(\omega) v^{2}(\omega) \tau(\omega) \rho\left(\omega^{2}\right),
$$

where

$$
C(\omega)=\frac{\hbar^{2} \omega^{2}}{k_{\mathrm{B}} T^{2}} n(\omega)[n(\omega)+1], \quad n(\omega)=n\left(\omega_{\nu}\right), \quad \nabla_{k} \omega_{\nu}=v(\omega), \quad \tau^{-1}(\omega)=\sum_{i} \tau_{i}^{-1}(\omega)
$$

Here $\tau_{i}$ is a single-phonon relaxation time related to $i$-th mechanism of the phonon scattering $[8,14], \nabla_{k} \omega_{\nu}$ is the velocity of the acoustic phonons.

One can obtain the background (i.e. "normal") temperature dependence of HCC $\lambda(T)$ including in equations (12) and (13) the main "usual" phonon scattering mechanisms [5,8]: $\tau_{1}^{-1}=A_{1} T \omega^{2} \exp (-\alpha / T)$ is the three-phonon colliding of acoustic phonons, $\tau_{2}^{-1}=A_{2} \omega^{4}$ is the point defect phonon scattering, $\tau_{3}^{-1}=A_{3}$ is the boundary phonon scattering. Here $A_{1}, A_{2}, A_{3}, \alpha$ are constants for a corresponding sample of the crystals $[5,8,13]$. Therefore, for $\tau^{-1}(\omega)$ according to $(13),(8)$ we obtain $(14)$

$$
\tau^{-1}(\omega)=\tau_{0}^{-1}(\omega)+\tau_{s}^{-1}(\omega),
$$

where, $\tau_{0}^{-1}=\tau_{1}^{-1}+\tau_{2}^{-2}+\tau_{3}^{-1}, \tau_{s}^{-1}=\tau_{\text {in }}^{-1}+\tau_{\mathrm{c}}^{-1}$.

The difficulties of calculations using equations (12), (13), even for the simple model under consideration, are well known $[5,8,14]$.

The results obtained are applied in the numerical analysis of the temperature behavior of the HCC near the structural phase transition $T_{\mathrm{C}}$ for $\mathrm{SrTiO}_{3}$ and $\mathrm{KDP}-$ type crystals (figure 1). The parameters of the soft mode $\omega_{q}(T)=D\left(q_{0}^{2}+q^{2}\right), \omega_{0}^{2}=$ $D^{2} q_{0}^{2}=r\left|T-T_{0}\right|$ in $\tau_{0}\left(A_{1}, A_{2}, A_{3}, \alpha\right), \tau_{\mathrm{c}}\left(\Phi=\Phi_{1}\right.$ for $T<T_{\mathrm{C}}$ and $\Phi=\Phi_{2}$ for $T>T_{\mathrm{C}}$ ) are obtained [8] and compared with the experimental data [2,3]. The numerical values of $T_{\mathrm{C}}, \Theta, \Phi\left(\Phi_{1}, \Phi_{2}\right), T_{0}\left(T_{0}^{\prime}\right)$ for $\mathrm{SrTiO}_{3}$ and KDP-type crystals are given in table 1 . Here value $T_{0}$ is determined according to $(7)$ as $\omega_{0}\left(T_{0}\right)=0$ and $\omega_{0}\left(T_{\mathrm{C}}\right)=\delta_{0}, T_{0}<T_{\mathrm{C}}, \omega_{0}\left(T_{0}^{\prime}\right)=0$ and $T_{0}^{\prime}>T_{\mathrm{C}}$.

Table 1. The numerical values of $T_{\mathrm{C}}, \Theta, \Phi\left(\Phi_{1}, \Phi_{2}\right), T_{0}\left(T_{0}^{\prime}\right)$ for $\mathrm{SrTiO}_{3}$ and $\mathrm{KDP}-$ type crystals.

\begin{tabular}{|l|c|c|c|c|c|c|}
\hline Crystal & $T_{\mathrm{C}}, \mathrm{K}$ & $T_{0}, \mathrm{~K}$ & $T_{0}^{\prime}, \mathrm{K}$ & $\Theta, \mathrm{K}$ & $\Phi_{1}^{2}, \mathrm{MeV}$ & $\Phi_{2}^{2}, \mathrm{MeV}$ \\
\hline $\mathrm{KH}_{2} \mathrm{PO}_{4}$ & 122 & 118 & 126 & 325 & 0.082 & 0.204 \\
\hline $\mathrm{KD}_{2} \mathrm{PO}_{4}$ & 220 & 216 & 224 & 323 & 0.096 & 0.083 \\
\hline $\mathrm{KH}_{2} \mathrm{AsO}_{4}$ & 96 & 92 & 100 & 298 & 0.162 & 0.356 \\
\hline $\mathrm{SrTiO}_{3}$ & 108 & 103 & - & 700 & - & 0.840 \\
\hline
\end{tabular}




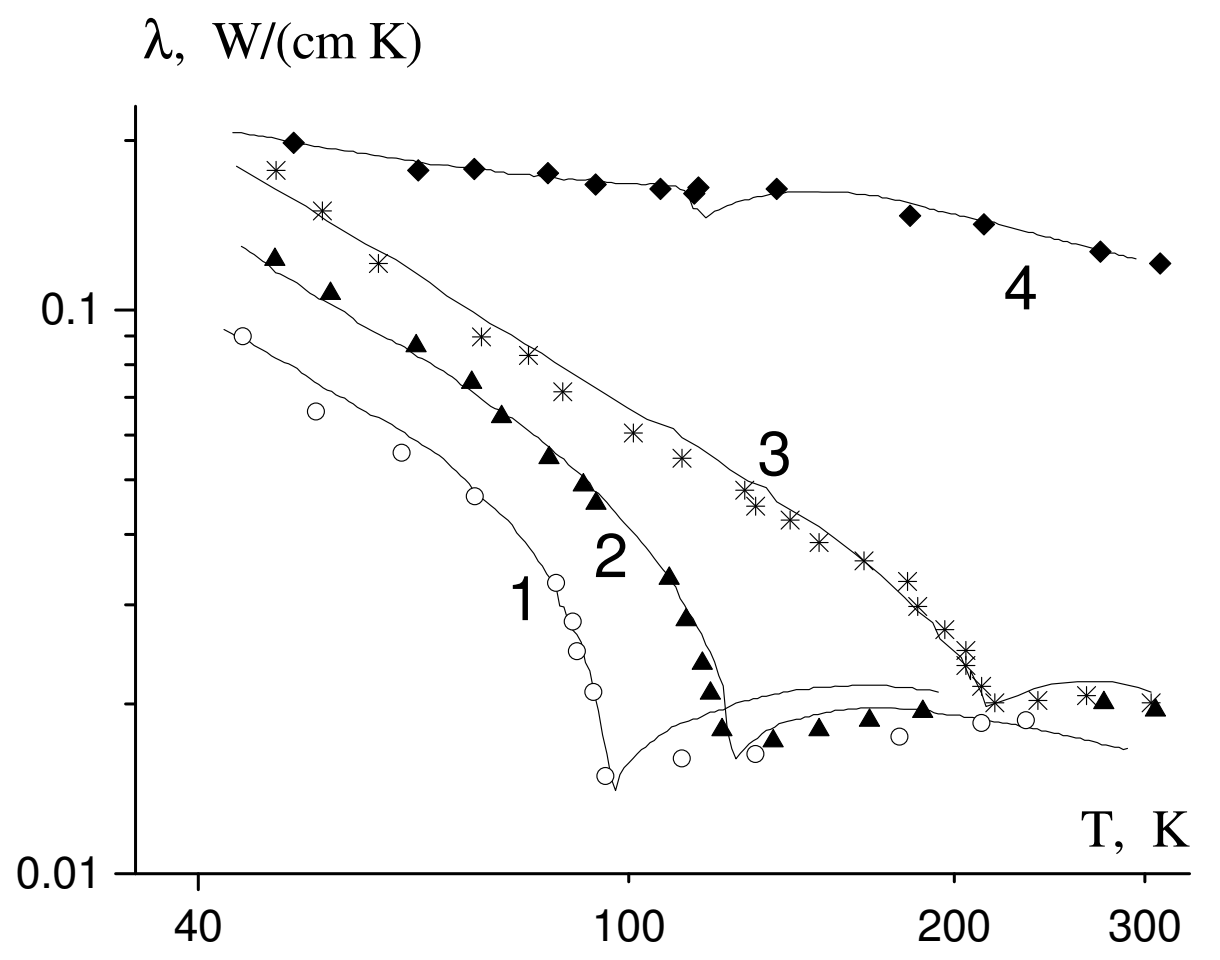

Figure 1. Temperature dependence of $\mathrm{HCC}$ for $\mathrm{KH}_{2} \mathrm{AsO}_{4}$ (1), $\mathrm{KH}_{2} \mathrm{PO}_{4}$ (2), $\mathrm{KD}_{2} \mathrm{PO}_{4}(3)$ [2] and $\mathrm{SrTiO}_{3}$ [3] crystals. Solid lines are the theory [8]. Peculiarities of the $\mathrm{C}$ - and E-type.

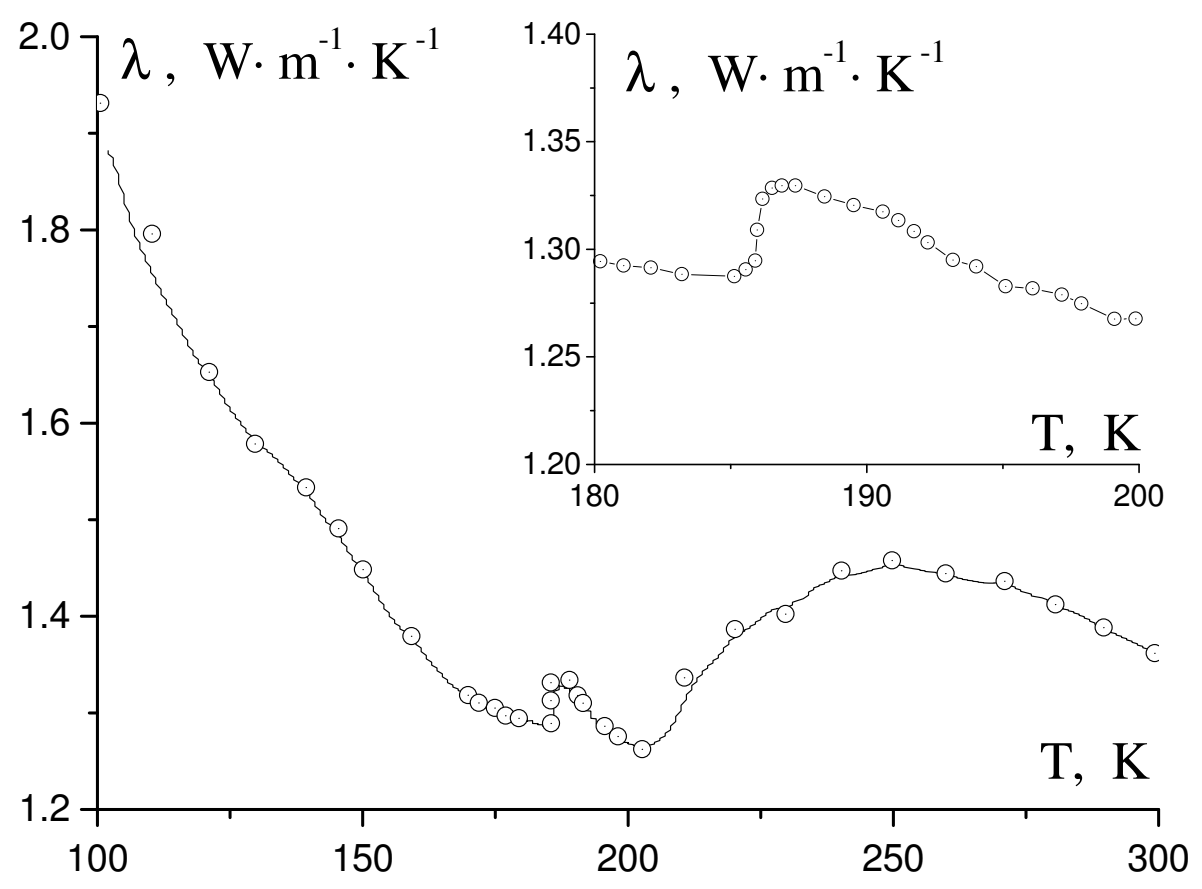

Figure 2. Temperature dependence of $\mathrm{HCC}$ for $\mathrm{Hg}_{2} \mathrm{Cl}_{2}$ crystal. Inset - the same in the vicinity of the phase transition point [1]. Peculiarity of the D-type. 
The agreement with experiments can be obtained due to adaptation of parameters $A_{1}, A_{2}, A_{3}, \alpha, \Phi$ (figure 1 ). It is seen, that the results of calculations are in a good accordance with the experimental data. Therefore, in the cases of $\mathrm{SrTiO}_{3}$ and KDP-type crystals, the quasi-elastic scattering is dominant.

The D-type anomaly of temperature dependence of HCC is presented at figure 2. This type of behavior is discussed in [19]. It may be shown that the peculiarities of this type are connected with the interference effect of different mechanisms of thermal resistance of a crystal. In the case of resonant mechanism of scattering we have $\lambda(T)$ behavior similar to the phonon-spectrometer in a dielectric crystal with paramagnetic ions [13]. The difficulties of HCC calculation, even for the simple model under consideration for

$$
\tau_{\text {eff }}^{-1}=\sum_{i} \tau_{i}^{-1}(\omega, k),
$$

where $\tau_{i}$ is a relaxation time of the $i$-th mechanism of the phonon scattering, are evident. It is clear, that the comprehensive explanation of the all types of HCC anomalies near $T_{\mathrm{C}}$ is a matter of the future more detailed theoretical study.

\section{Concluding remarks}

A general formula is obtained for the critical scattering of phonon by soft-phonon mode and central peak. It is shown that the quasi-elastic scattering becomes dominant over the inelastic scattering at least for temperatures close to $T_{\mathrm{C}}$. On the curve of the thermal conductivity $\lambda(T)$ near $T_{\mathrm{C}}(\mathrm{a}),(\mathrm{b}),(\mathrm{c}),(\mathrm{d})$ and (e)-type anomalies may be observed. In figures 1 and 2 the peculiarities (c), (d), and (e)-types in comparison with the corresponding experiments are presented. The peculiarities of another type and its classification on the whole, require more accurate consideration.

\section{References}

1. Strukov B.A., Belov A.A. // Phase transitions, 1994, vol. 51, p. 175.

2. Suemnne Y.J. // Phys. Soc. Jap., 1967, vol. 22, p. 735.

3. Steigmeier E.F. // Phys. Rev., 1968, vol. 168, p. 523.

4. Inone M.J. // J. Phys. Soc. Jap., 1969, vol. 26, p. 420.

5. Nettlton R.E. // Ferroelectrics, 1970, vol. 1, p. 87.

6. Balagurov B.J. // JETP, 1971, vol. 61, p. 1627.

7. Altukhov V.I. // Phys. Stat. Sol. (b), 1979, vol. 93, p. K115.

8. Altukhov V.I. // Izv. AN Est. SSR, ser. fiz. i mat., 1980, vol. 29, p. 46.

9. Mermelstein M.D., Cummins H.Z. // Phys. Rev. B, 1977, vol. 16, p. 2177.

10. Aksenov V.I., Plakida N.M. The Neutron Scattering in Ferroelectrics. Moscow, Energoatomizdat, 1984 (in Russian).

11. Kobayashi K.K. // J. Phys. Soc. Jap., 1968, vol. 24, p. 497.

12. Iolin E.M. // Fiz. Tverd. Tela, 1970, vol. 12, p. 1159.

13. Altukhov V.I., Zavt G.S. // Fiz. Tverd. Tela, 1977, vol. 19, p. 1057.

14. Kascheev K.N. // Izv. AN Latv. SSR, ser.fiz. i tekh. nauk, 1985, vol. 6, p. 20. 
15. Kubo R. Thermodynamics of Irreversible Processes. Moscow, IL, 1962 (in Russian).

16. Altukhov V.I. // Phys. Stat. Sol. (b), 1974, vol. 64, p. 403.

17. Altukhov V.I., Zavt G.S. // Phys. Stat. Sol. (b), 1974, vol. 65, p. 83.

18. Pytte E. // Phys. Rev. B, 1970, vol. 1, p. 924.

19. Strukov B.A., Belov A.A., Altukhov V.I. // Ferroelectrics, 1994, vol. 159, p. 25.

20. Strukov B.A., Belov A.A. // Ferroelectrics, 1992, vol. 126, p. 299.

21. Altukhov V.I. D. Sci. Thesis. Moscow, Moscow State University, 1990.

22. Levanyuk A.P., Minyukov S.A., Vallade M. // J. Phys. (France), 1992, vol. 2, p. 1949.

23. Levanyuk A.P., Sigov A.S. Defects and Structural Phase Transition. New-York, Gordon and Breach, 1986.

\title{
Критичне розсіяння фононів і особливості явищ переносу в сегнетоелектричному кристалі
}

\author{
В.І.Алтухов ${ }^{1}$, Б.А.Струков ${ }^{2}$ \\ 1 Північно-кавказький державний технічний університет, \\ Росія, 355000 Ставрополь \\ 2 Московський державний університет, Росія, 119992 Москва
}

Отримано 6 серпня 2002p.

Представлено огляд теоретичних досліджень критичного розсіяння фононів і аномального переносу тепла поблизу температури фазового переходу $T_{\mathrm{c}}$. Обговорюється інтерпретація і кількісний підхід до опису аномальної температурної залежності $\lambda(T)$ поблизу $T_{\mathrm{c}}$.

Ключові слова: сегнетоелектрики, фазові переходи, критичне розсіяння фононів, теорія переносу тепла, м'яка фононна мода, центральний пік

PACS: 77.84.-s, 77.80.Bh, 63.20.-e, 66.70. $+f$ 